\title{
Distribución espacio-temporal de Diatraea spp. (Lepidoptera: Crambidae) en el valle del río Cauca, Colombia
}

\author{
Spatial and temporal distribution of Diatraea spp. (Lepidoptera: Crambidae) in the Cauca River Valley, Colombia
}

\author{
SAMY ANDRÉS LEYTON-FLOR ${ }^{1}$, MARISOL GORDILLO², PAULA A. GONZÁLEZ ${ }^{3}$, \\ JOHANN A. OSPINA ${ }^{4}$ y GERMÁN VARGAS 5
}

\begin{abstract}
Resumen: En el valle del río Cauca se ha incrementado el daño por parte de los barrenadores del tallo, Diatraea spp., en la caña de azúcar, debido a dos nuevas especies, D. tabernella y D. busckella, que se sumaron a las ya conocidas $D$. saccharalis y $D$. indigenella. Se estudió la bioecología de la plaga y de sus enemigos naturales para ajustar los planes de manejo, que consisten principalmente en control biológico. Se realizó el seguimiento de la plaga en un ciclo de cultivo, en nueve campos distribuidos por todo el valle del río Cauca. El muestreo se hizo mediante una cuadrícula de 30x30 metros y 50 puntos georreferenciados en cada campo. Se registró el número de tallos afectados y del número de entrenudos barrenados por tallo. En el exterior de la cuadrícula se recolectaron huevos y larvas de la plaga mediante un esfuerzo de muestreo de una hora-hombre. La zona norte presentó los mayores niveles de daño y el predominio de $D$. tabernella y D. busckella. Los parasitismos de huevo por Trichogramma exiguum llegaron hasta el $98 \%$ y Cotesia flavipes fue el parasitoide de larvas más abundante. Hubo correlaciones positivas entre el porcentaje de tallos con daño (\%TD) y la edad de la caña, y entre el número de larvas y las larvas parasitadas. El análisis espacial del \%TD evidenció una distribución agregada, concentrándose en los bordes de la cuadrícula de muestreo. El esquema de manejo debe considerar liberaciones de insectos benéficos en las etapas avanzadas del cultivo y dirigido a los focos de infestación.
\end{abstract}

Palabras clave: D. tabernella, D. busckella, D. saccharalis, D. indigenella, parasitoides.

\begin{abstract}
Sugarcane stem borer (Diatraea spp) damage has increased in the Cauca River Valley (CRV) due to two new species, $D$. tabernella and $D$. busckella, which joined the two already known species, D. saccharalis and $D$. indigenella . A study of the bioecology of these pests and their natural enemies is presented in order to increase the effectiveness of pest management, which consists primarily of biological control. An assessment of the extent of pest damage was completed during a crop cycle in nine fields distributed along the CRV. Sampling was done in a 30x30 meter grid and 50 points were geo-referenced in each field. A registry was made of the number of damaged stalks and the number of bored internodes per stalk. Outside the grid, eggs and larvae of the pests were collected by means of a one man-hour sampling effort. The northern CRV showed the greatest levels of pest injury and a predominance of $D$. tabernella and D. busckella. Egg parasitism by Trichogramma exiguum reached levels of $98 \%$, whereas Cotesia flavipes was the most abundant larval parasitoid. There were positive correlations between the percent of damaged stalks (\%TD) and crop age, and between the number of larvae and parasitized larvae. A spatial analysis of \%TD showed an aggregated distribution concentrated toward the edge of the grid. Pest management protocols should be differentiated by region within the CRV in order to prioritize areas of greatest infestation.
\end{abstract}

Key words: D. tabernella, D. busckella, D. saccharalis, D. indigenella, parasitoids.

\section{Introducción}

Los barrenadores del tallo Diatraea spp. constituyen las plagas de mayor importancia en el cultivo de la caña de azúcar en América y de las cuales se tiene conocimiento de un total de 41 especies (Solis y Metz 2016). En el caso de Colombia se tiene registro de al menos seis especies; sin embargo, este registro requiere de una mayor actualización (Solis y Metz 2016). En el valle del río Cauca se tenía el registro de Diatraea saccharalis (F.) y $D$. indigenella Dyar y Heinrich, pero recientemente se han detectado nuevas especies como $D$. $t a-$ bernella Dyar y D. busckella Dyar y Heinrich, causando el incremento del daño en la región y en particular de las zonas norte y centro, respectivamente (Gómez y Vargas 2014).

Los barrenadores del tallo son plagas que atacan el cultivo de la caña de azúcar desde germinación, causando el síntoma de corazón muerto, hasta la etapa de cosecha, en donde el daño de observa en las galerías formadas por el insecto. Luego el insecto empupa en el mismo tallo para dar paso a la emergencia del estado adulto, que es una polilla de hábito nocturno y que deposita sus huevos especialmente en el envés de las hojas de la caña (Vargas et al. 2015a). Estos mismos autores señalan que el efecto de la plaga en la pérdida de peso del cultivo puede llegar a ser de $0,83 \%$ por cada unidad porcentual de entrenudos barrenados.

De acuerdo con Vargas (2015) en el valle del río Cauca el seguimiento de las poblaciones de la plaga se realiza mediante la evaluación del porcentaje de entrenudos barrenados al momento de la cosecha con la toma de 100 tallos al azar por lote comercial, información que permite planificar el manejo mediante la liberación de enemigos naturales durante el ciclo de cultivo consecutivo, es decir siguiente a la cosecha (una siembra de caña puede representar entre 3, 5 o incluso más cortes consecutivos que se conocen como socas). Este

\footnotetext{
${ }^{1}$ Administrador Ambiental, M. Sc., Estudiante, Universidad Autónoma de Occidente, Cali, Colombia, leyton311@hotmail.com. ${ }^{2}$ Estadística, Ph. D., Docente Universidad Autónoma de Occidente, Cali, Colombia, mgordillo@uao.edu.co. ${ }^{3}$ Licenciada en Matemáticas, Ph. D., Docente Universidad Autónoma de Occidente, Cali, Colombia, pagonzalez@uao.edu.co. ${ }^{4}$ Estadístico, M. Sc., Docente Universidad Autónoma de Occidente, Cali, Colombia,jaospina@uao.edu. co. ${ }^{5}$ Ing. Agrónomo, Ph. D., Entomólogo Cenicaña, Cali, Colombia, gavargas@cenicana.org. Autor para correspondencia: Germán Vargas. Ing. Agrónomo, Ph. D., Entomólogo Cenicaña, Cali, Colombia, gavargas@cenicana.org.
} 
esquema de control biológico ha sido la principal estrategia para el manejo de la plaga y hasta el momento ha permitido mantener los niveles de daño promedio de la industria azucarera por debajo de niveles del $5 \%$ de entrenudos barrenados por cerca de tres décadas (Gómez y Vargas 2014). Al respecto, Vargas et al. (2005) reportan un brote por la plaga en 2005 en donde el uso de la geoestadística fue una herramienta utilizada en el manejo de los barrenadores. Estos autores utilizaron la información del porcentaje de entrenudos barrenados por la plaga para generar un mapa de isolíneas de daño aplicando los métodos de interpolación de "kriging" o de extensión del programa ArcGIS $\AA$, que permitió detectar una zona de alta infestación entre El Cerrito y Palmira (con niveles de daño entre 6 y $8 \%$ de entrenudos barrenados), y un sector de afectación intermedia entre Cartago y Obando (con niveles de daño entre 4 y $6 \%$ de entrenudos barrenados). El esfuerzo coordinado de los diferentes ingenios y agricultores llevaron a que en un tiempo de un año se tuvieran indicadores inferiores de daño en las diferentes zonas detectadas como problema, con niveles de daño entre 2 y $4 \%$ entre El Cerrito y Palmira, y entre 0 y $2 \%$ entre Cartago y Obando (Gómez y Cadena 2007).

Dentro de la fauna benéfica que afecta los diferentes estados de la plaga en la región están parasitoides de huevo, especialmente las avispitas Trichogramma exiguum Pinto y Platner (Hymenoptera: Trichogrammatidae), mientras que en el caso del estado larval se tienen las moscas Lydella minense (Townsend) y Billaea claripalpis (Wulp) (Diptera: Tachinidae), que se producen masivamente para su liberación en los campos, y la mosca silvestre Genea jaynesi (Rondani) (Diptera: Tachinidae), de gran importancia en el control biológico natural de la plaga, pero sin la posibilidad de realizar liberaciones dado que no se ha podido desarrollar su cría masiva en laboratorio (Vargas et al. 2006). Los parasitismos por los taquínidos pueden llegar hasta valores promedios de $28 \%$ (Trejos et al. 1986; Vargas et al. 2015b) y se consideran de vital importancia en la regulación de la plaga. Además, se tiene a Cotesia flavipes Cameron (Hymenoptera: Braconidae), parasitoide de larvas de uso masivo en Centroamérica y Brasil (Badilla 2002; Postali et al. 2010), que recientemente se ha estado liberando en el valle del río Cauca, inicialmente en la zona norte de la región, y a partir de lo cual se está dando un proceso gradual de adaptación en todo el valle del río Cauca (Vargas et al. 2015b).

Los brotes de la plaga presentados en las zonas norte y centro del valle del río Cauca, asociados con la detección de nuevas especies de la plaga, han planteado inquietudes acerca de si los métodos de control utilizados convencionalmente en el manejo de la plaga siguen siendo adecuados y sí existen oportunidades de mejorar lo relacionado con el monitoreo de las poblaciones de la plaga y la acción de sus enemigos naturales (Vargas et al. 2013).

Es de señalar que no existen referencias acerca de la dinámica poblacional del complejo de especies de Diatraea presente en el valle del río Cauca con relación a la edad del cultivo de la caña de azúcar. Al respecto, Vargas et al. (2015a) observaron que la oviposición de $D$. indigenella es muy escasa en campos con edades inferiores a los seis meses de edad mientras que se incrementa a partir de los siete meses, lo que coincide con una mayor población de larvas de $D$. indigenella a partir de este periodo y que contrasta con una mayor población de larvas de $D$. saccharalis en edades inferiores y que disminuye al avanzar la madurez del cultivo (Gómez y
Vargas 2014). Sin embargo, estos autores no brindan información acerca del comportamiento de D. tabernella y/o $D$. busckella, causantes del brote de la plaga en la actualidad. De acuerdo con lo anterior el objetivo de este trabajo es estudiar la distribución del complejo de barrenadores de la caña de azúcar teniendo en cuenta su distribución espacial y su abundancia temporal, esto a través de un ciclo de cultivo y abarcando áreas diferentes del valle del río Cauca en razón a la distribución desigual de estas especies en la región (Vargas et al. 2018). Lo anterior se complementa con el registro de sus enemigos naturales a nivel de parasitoides de huevo y de larva en los diferentes sitios muestreados. Se espera que lo anterior permita ajustar los métodos de monitoreo y las acciones de control biológico, teniendo en cuenta que este es el principal método de manejo utilizado en la actualidad.

\section{Materiales y métodos}

Sitios de muestreo. Dado que no se disponía de antecedentes que justificaran una distancia de evaluación ni el número de muestras a tomar, fue necesario establecer el valor óptimo, dependiendo del grado de variación espacial y el error de muestreo, para ello se realizó un muestreo preliminar en tres suertes (campos comerciales) con la misma variedad de caña sembrada (CC 85-92), entre 11 y 12 meses de edad y localizados en diferentes partes del valle del río Cauca. Uno de los campos estuvo en la zona norte (municipio de Cartago, Valle del Cauca, Ingenio Risaralda, Hacienda La Holanda, suerte 9, 0443'771'N, 7557.946"O), otro en el centro (municipio de Tuluá, Valle del Cauca Ingenio Sancarlos, Hacienda Argelia,

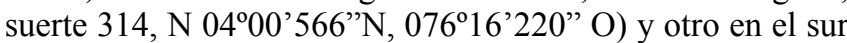
(municipio de Padilla, Cauca, Ingenio Incauca, Hacienda San Fernando Sur, suerte 11, 03 ${ }^{\circ} 16^{\prime} 021^{\prime \prime}$ N, 076120'763'O). En los diferentes campos se estableció una cuadrícula de $120 \mathrm{x}$ $120 \mathrm{~m}$ (1,44 ha), con una distancia de 10 metros entre puntos, para un total de 140 puntos por cuadrícula. Los puntos fueron georeferenciados usando GPS y sirvieron para tomar una cepa de caña en donde se contabilizaron el número de tallos y el número de tallos con síntomas de ataque por Diatraea spp., lo que permitió estimar el porcentaje de tallos atacados (infestación). Para determinar la distancia mínima entre puntos (m) y el tamaño de la cuadrícula (número de puntos a utilizar) que permitan encontrar el daño por Diatraea se estimó la varianza dentro de cada cuadrícula, y se utilizó la siguiente expresión:

$$
n=\frac{Z^{2} \alpha / 2 \hat{\sigma}^{2}}{d 2}
$$

donde, $Z$ es el punto porcentual para un nivel de significancia dado $(\alpha), \hat{\sigma}^{2}$ es la varianza estimada, siendo la más grande encontrada entre todas las distancias y en las zonas, y $d$ es el error de muestreo o tolerancia. De acuerdo con lo anterior, se obtuvo que con un margen de error de 0,0525 metros y un nivel de significancia de 0,1 ; el número de puntos debe ser de 50, distanciados cada 30 metros (cuadrícula). Este criterio fue utilizado para el seguimiento del daño por la plaga durante un ciclo de cultivo, en nueve campos distribuidos a lo largo del valle del río Cauca.

Los campos fueron seleccionados con base en que estuvieran distribuidos a lo largo del valle del río Cauca, que al momento de iniciar las observaciones tuvieran una edad in- 
ferior a un mes desde la cosecha anterior (sólo se tuvieron en cuenta socas y no campos recién renovados), que estuvieran sembrados con la misma variedad de caña de azúcar (CC 8592) y con antecedentes de ataque por la plaga (Tabla 1).

Establecimiento de los puntos de muestreo en las zonas de evaluación. Los puntos de las cuadrículas fueron georreferenciados y rotulados para su posterior ubicación en campo. En los puntos se observó el número de tallos por cepa y el número de tallos con síntomas de ataque por Diatraea spp. Los muestreos se realizaron a partir del primer mes de edad de la caña y seguidamente a los 3, 5, 7, 9 y 11 meses de edad del cultivo. Las observaciones de tallos afectados por cepa se hicieron sin destrucción de los mismos y durante todo el periodo de evaluaciones. Por otro lado, teniendo en cuenta que después del cuarto mes ya se presentan entrenudos en los tallos y que para la correcta observación del daño por la plaga en los entrenudos es necesario el corte de los tallos, las evaluaciones de entrenudos barrenados se realizaron sólo a los 5 y 11 meses de edad rajando la caña para contar los entrenudos totales por tallo y aquellos con síntomas de ataque por la plaga. Lo anterior se realizó tomando un tallo por cada cepa, es decir un total de 50 tallos por lote evaluado. Las evaluaciones se realizaron entre octubre 30 de 2014 y septiembre 22 de 2015.

Evaluación del daño de Diatraea spp. durante un ciclo de cultivo de caña de azúcar. Para el seguimiento del daño por la plaga se tuvieron en cuenta las siguientes variables: el número de tallos por planta de caña o cepa y el número de tallos con daño por cepa, que permitieron estimar el porcentaje de tallos con daño (TD \%):

$$
\mathrm{TD} \%=\frac{\text { Número de tallos daño }}{\text { Número total de tallos }} \times 100
$$

Además, a los 5 y 11 meses de edad, se cuantificó el total de entrenudos por tallo y el número de entrenudos con daño por Diatraea spp., esto mediante el corte de un tallo por cepa. A dicho tallo se le retiró su corteza en dos cortes longitudinales dejando caras opuestas y expuestas del tallo con el fin de observar mejor los daños internos por la plaga. Lo anterior permitió estimar el porcentaje de entrenudos barrenados o intensidad de infestación (II \%).

$$
\text { II \% }=\frac{\text { Número de entrenudos con daño }}{\text { Número total de entrenudos }} \times 100
$$

Captura de larvas y huevos de Diatraea spp. Se recolectaron larvas y posturas de Diatraea spp. en una zona aledaña a la cuadrícula de muestreo, con iguales características de edad y variedad y con la misma frecuencia de la observación del daño. No se realizaron recolecciones en la cuadrícula de muestreo con el propósito de no interferir con la dinámica poblacional de los insectos. Se destinó un esfuerzo de muestreo consistente en una hora-hombre por sitio tanto para la captura de larvas y huevos. La identificación de las larvas se realizó en el laboratorio de entomología de Cenicaña. Con relación al estado de huevo la recolección de estos se da principalmente del envés de las hojas y, hasta la fecha, no existe forma de diferenciar los huevos entre las diferentes especies de Diatraea por lo que se manejó a nivel de Diatraea spp. En lo referente a los parasitoides de huevos, la identificación de las avispitas se realizó con base en el reconocimiento de especies de Trichogramma asociadas con caña de azúcar en Colombia (Gómez et al. 1996). Además, de acuerdo con la coloración de las larvas se realizó una identificación preliminar, que sirvió para asignar la identidad del hospedante en el caso de la aparición de los parasitoides, ya fueran estas moscas taquínidas o avispitas de C. flavipes (Vargas 2015). La corroboración de la identidad de la especie fue realizada con base en las características del estado de pupa y de la genitalia del macho en el estado adulto (Solis y Metz 2016; Vargas et al. 2018).

Análisis geoestadístico, estimación de semivariogramas y "krigeado" y análisis estadístico. Por medio del análisis geoestadístico se cuantificó y caracterizó la variabilidad espacial de Diatraea spp. Esta técnica ha sido ampliamente utilizada en la llamada agricultura de precisión, realizando aplicaciones de patrones espaciales en distintas direcciones del espacio (Oliver 2013). Las predicciones en el espacio se pueden realizar mediante modelos "kriging" (Gallardo 2006). Se trata entonces de aplicar una técnica de predicción, y para esto, se necesita conocer la estructura de autocorrelación para cualquier posible distancia entre posiciones. El "kriging" encierra un conjunto de métodos de predicción espacial que se fundamentan en la minimización del error cuadrático medio de predicción (Giraldo 2002). La forma de describir la varia-

Tabla 1. Campos comerciales seleccionados para el estudio de la distribución espacio-temporal de las poblaciones de Diatraea spp. en diferentes zonas del valle del río Cauca y durante un ciclo de cultivo entre octubre 30 de 2014

\begin{tabular}{|c|c|c|c|c|}
\hline Zona & Ingenio & Municipio & Hacienda, suerte & Coordenadas \\
\hline \multirow{3}{*}{ Norte } & Risaralda & Viterbo, Caldas & Garona, 3 & 0459'252”N $075^{\circ} 51^{\prime} 703^{\prime \prime} \mathrm{O}$ \\
\hline & Risaralda & Viterbo, Caldas & Las Villas, 9 & $04^{\circ} 56^{\prime} 180^{\prime \prime} \mathrm{N} \quad 075^{\circ} 53.569^{\prime \prime} \mathrm{O}$ \\
\hline & Risaralda & Viterbo, Caldas & Santa Mónica, 6 & $04^{\circ} 35^{\prime} 492^{\prime \prime} \mathrm{N} 076^{\circ} 03^{\prime} 257^{\prime \prime} \mathrm{O}$ \\
\hline \multirow{3}{*}{ Centro } & Sancarlos & Tuluá, Valle & La Argelia, 420A & $04^{\circ} 01^{\prime} 242^{\prime \prime} \mathrm{N} \quad 076^{\circ} 17^{\prime} 119^{\prime \prime} \mathrm{O}$ \\
\hline & Providencia & Yumbo, Valle & Villa Inés, 7A & $03^{\circ} 37^{\prime} 998^{\prime \prime} \mathrm{N} \quad 076^{\circ} 26^{\prime} 575^{\prime \prime} \mathrm{O}$ \\
\hline & Providencia & Yumbo, Valle & Samaria, 162A & 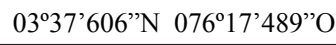 \\
\hline \multirow{3}{*}{ Sur } & Mayagüez & Pradera, Valle & Venecia, 93A & $03^{\circ} 25^{\prime} 978^{\prime \prime} \mathrm{N} \quad 076^{\circ} 18^{\prime} 302^{\prime \prime} \mathrm{O}$ \\
\hline & Incauca & Padilla, Cauca & San Fernando Sur, 31 & $03^{\circ} 16^{\prime} 368^{\prime \prime} \mathrm{N} \quad 076^{\circ} 20^{\prime} 443^{\prime \prime} \mathrm{O}$ \\
\hline & Incauca & Jamundí, Valle & La Isla, 2 & $03^{\circ} 15^{\prime} 288^{\prime \prime} \mathrm{N} \quad 076^{\circ} 28^{\prime} 162^{\prime \prime} \mathrm{O}$ \\
\hline
\end{tabular}
y septiembre 22 de 2015. 
ción del proceso aleatorio subyacente se realiza por medio de variables regionalizadas, es decir, variables medidas en el espacio de forma que presenten una estructura de correlación, suponiendo que variables cercanas podrían estar autocorrelacionadas se describe la relación entre pares de posiciones por medio de la covarianza espacial (Oliver 2013).

El semivariograma muestral fue calculado sólo para algunas distancias promedios particulares, por lo tanto fue necesario ajustar por medio de modelos que permitieran generalizar la dependencia espacial para cualquier distancia. En cada zona de estudio se consideró un semivariograma experimental a partir de los registros de porcentaje de tallos con daño (TD \%) y de la Intensidad de Infestación (II \%), por medio de la siguiente ecuación:

$$
\hat{\gamma}(h)=\frac{1}{2 m(h)} \sum_{i=1}^{m(h)}\left\{z\left(x_{i}\right)-z\left(x_{i}+h\right)\right\}^{2}
$$

Donde $Z\left(x_{i}\right)$ y $Z\left(x_{i}+h\right)$ son los valores de $Z$ para las posiciones $x_{i}{ }^{y} x_{i+(h)} ; m(h)$ es el número de comparaciones pareadas para el rezago $h$ (Webster y Oliver 2007).

Para la construcción de los mapas de daño no es suficiente el cálculo de los semivariogramas, es necesario ajustar un modelo que permita cuantificar el ajuste del modelo, entre los que se encuentra gaussianos, esféricos, lineales y exponenciales. Tanto el análisis exploratorio como la modelación de los Semivariogramas y mapas "kriging" fueron elaborados en el paquete estadístico R (R Core Team 2013).

La variable número de larvas fue comparada con el número de larvas parasitadas por diferentes parasitoides usando un análisis de regresión lineal, de igual manera la relación entre la edad de la caña y los porcentajes de tallos atacados fueron correlacionados para cada una de las zonas, utilizando en ambos casos el paquete estadístico Minitab (Minitab 2017).

\section{Resultados y discusión}

Población de Diatraea spp. (huevos y larvas). Durante todo el ciclo de cultivo y en las tres zonas de evaluación se recolectaron un total 687 larvas de Diatraea spp., en donde la zona norte mostró la mayor población, seguida en su orden por las zonas centro y sur (Fig. 1A). En el norte las capturas fluctuaron entre 40 larvas (3er. mes) y 100 larvas $\left(7^{\circ} \mathrm{mes}\right)$, con una captura de 60 larvas al $11^{\circ}$ mes. En el caso del centro se observó un incremento sostenido del número de larvas desde el tercer mes, pero los niveles máximos de captura ocurrieron al $9^{\circ}$ mes con 40 larvas. Por su parte, en el sur los niveles no mostraron una tendencia definida y fluctuaron alrededor de 20 larvas (Fig. 1A).

De forma correspondiente en cuanto al número de huevos encontrados la zona norte presentó la mayor cantidad, con la tendencia a incrementarse a partir del tercer mes de edad de la caña (Fig. 1B). Aunque en este estado de la plaga no es posible identificar con precisión la especie de Diatraea, se puede considerar que estas posturas pertenecen a $D$. $t a-$ bernella y D. busckella, ya que son las dos únicas especies que se encontraron en las parcelas de la zona norte del valle del río Cauca (Fig. 2B). Lima y Gomes (2003) registraron huevos de $D$. saccharalis desde el primer mes del cultivo en campos en el norte del estado de Río de Janeiro en Brasil. Por su parte, Gómez y Vargas (2014) estudiaron la población de $D$. indigenella desde los dos meses de edad hasta cosecha y sólo encontraron huevos a partir de los siete meses de edad, tiempo a partir del cual se evidenció un crecimiento marcado que sólo se presentó en el presente trabajo para la zona norte. Al respecto, Vargas et al. (2015b) reportaron la presencia de huevos de $D$. tabernella en campos en el norte del valle del río Cauca en edades entre 1 y 1,5 meses; sin embargo, el presente trabajo reporta la presencia de huevos de la plaga desde el primer mes sólo para la zona sur, mientras que hubo huevos en todas las zonas a partir del tercer mes.

A nivel del seguimiento a las larvas, D. tabernella fue encontrada sólo en la zona norte, mientras que $D$. busckella en las tres zonas; por su parte $D$. saccharalis y $D$. indigenella sólo se presentaron en los campos de las zonas centro y sur (Fig. 2). D. tabernella y D. busckella son las más abundantes en las tres suertes ubicadas en la zona norte, durante todas las etapas de desarrollo de la caña y con capturas totales por encima de 20 larvas durante casi todo el tiempo. Ambas especies tienen su pico máximo en el séptimo mes de edad. Sin embargo, la población de $D$. tabernella comienza a disminuir en las etapas finales del cultivo mientras que D. busckella no presenta fluctuaciones prominentes. Por su parte, en las zonas centro y sur las capturas difícilmente llegaron al nivel de 20 larvas totales, esto ocurrió específicamente en el caso de D. busckella en la zona centro a partir del quinto mes de edad de la caña, mientras que en la zona sur las mayores capturas estuvieron representadas por D. busckella hacia el mes 11 de edad de la caña.

Las investigaciones citadas por Gómez y Vargas (2014) reportan que en los años noventa, en la zona centro del valle del río Cauca (Ingenio Pichichí), D. saccharalis era más abundante en los primeros seis meses del cultivo, mientras que $D$. indigenella ocurría en las etapas de madurez del cultivo. En el presente trabajo $D$. indigenella presentó un com-

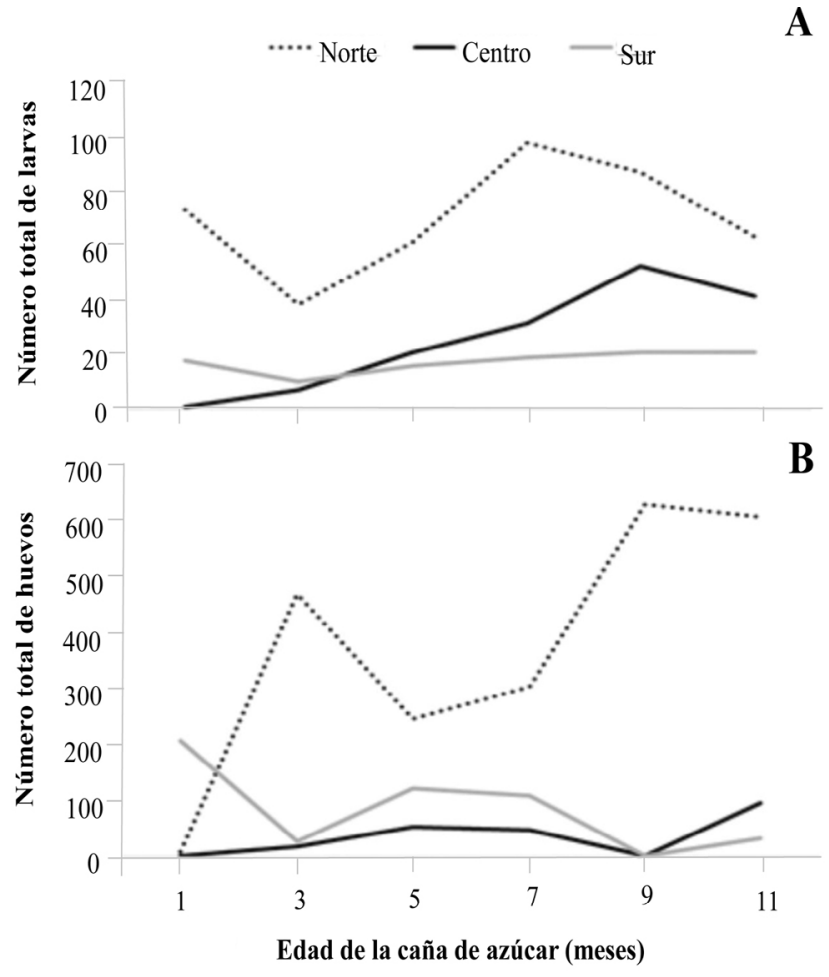

Figura 1. Número total de larvas (A) y huevos (B) en tres campos localizados en cada una de tres zonas (norte, centro y sur) del valle del río Cauca y durante un ciclo de cultivo de la caña de azúcar (1 - 11 meses). 


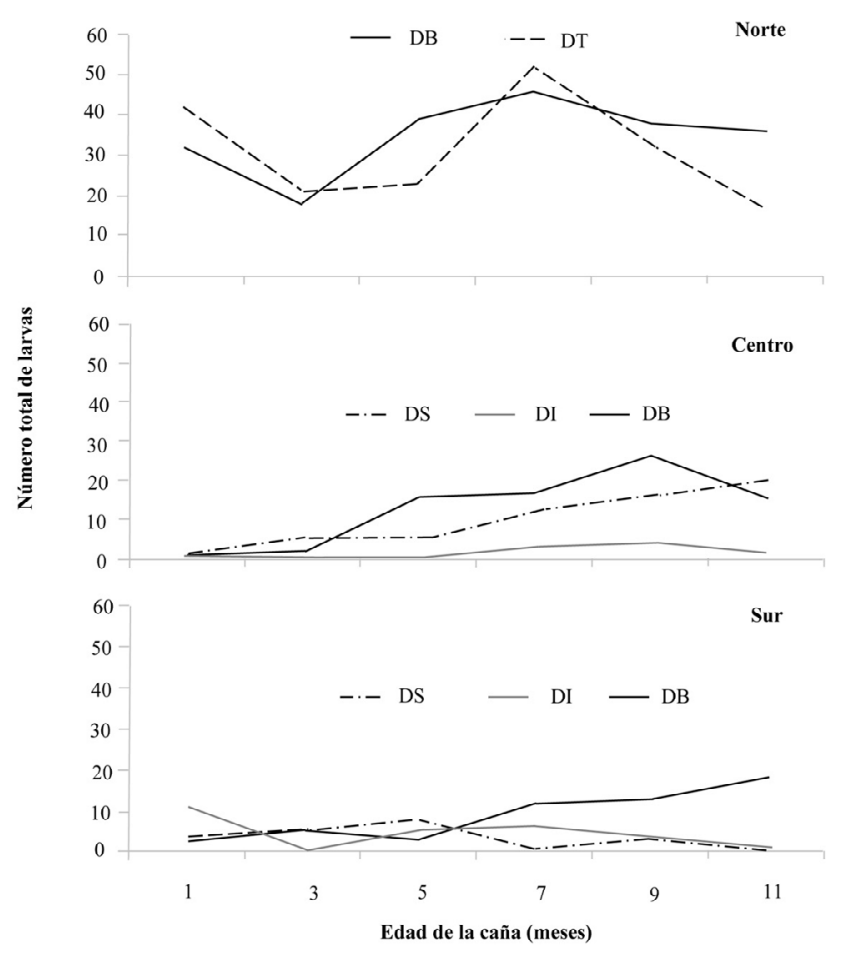

Figura 2. Número total de larvas recolectadas de Diatraea spp. en tres campos localizados en cada una de tres zonas del valle del río Cauca (Norte, Centro y Sur) durante un ciclo de cultivo de la caña de azúcar (1 - 11 meses). DS: Diatraea saccharalis, DI: D. indigenella, DB: D. busckella y DT: D. tabernella.

portamiento similar al reportado por Gomez y Vargas (2014); por su parte, el comportamiento de $D$. saccharalis presenta un patrón diferente al reportado por estos autores ya que esta especie fue más abundante en etapas maduras de la caña. Lo anterior indicaría que los comportamientos de estas especies pueden ser modificados por aspectos que no son posibles de determinar con la información obtenida en este estudio, pero que resaltan la importancia de mantener en ejercicio actividades de monitoreo de la abundancia de estas especies en los diferentes campos cultivados, con el fin de ajustar los planes de manejo.

Parasitismo por enemigos naturales en larvas y huevos de Diatraea spp. Se encontró una correlación lineal positiva entre el número de larvas y el número de larvas parasitadas por los diferentes parasitoides de larvas $(\mathrm{F}=82,7$; g.l. $=1$; $\mathrm{P}<$ 0,$\left.001 ; R^{2}=0,85\right)$, sugiriendo que a medida que exista mayor cantidad de huéspedes en el estado de larva habrá un aumento de los parasitoides, es decir una interdependencia poblacional entre enemigos naturales y hospedantes. En la Figura 3 se muestra el número de larvas totales encontradas parasitadas por diferentes enemigos naturales, donde $C$. flavipes aparece desde el quinto mes de edad del cultivo y es el parasitoide de mayor presencia en los últimos meses de desarrollo de la caña en la zona norte. $C$. flavipes se presentó de manera escasa a los 11 meses de edad en la zona centro, mientras que no se reportó en la zona sur. En general, los parasitismos en las larvas sólo se hicieron evidentes a partir del tercer mes de edad de la caña, donde los taquínidos $L$. minense y $G$. jaynesi fueron los primeros en aparecer, siendo G. jaynesi el más abundante de los taquínidos en las tres zonas. Por su parte, Billaea claripalpis sólo se evidenció en la zona sur al $11^{\circ}$ mes de muestreo.

El complejo de parasitoides usados en el programa de control biológico en el valle del río Cauca, conformado por $L$. minense, $B$. claripalpis, $C$. flavipes y $T$. exiguum, ha tenido un relativo éxito para reducir las poblaciones de Diatraea spp. en cultivos de caña establecidos en otros países de América, sin embargo no se tiene registro de algún otro país que tenga dentro de sus planes de manejo el uso de todos ellos juntos, como es el caso colombiano (Vargas 2015). Las investigaciones por Badilla (2002) y Salazar et al. (2003) demuestran la eficacia del parasitoide de larvas $C$. flavipes en la disminución del daño debido a su amplia capacidad de búsqueda y adaptación a las diferentes condiciones climáticas de Costa Rica. Sin embargo, Rodríguez et al. (2004) afirmaron que $C$. flavipes no era muy efectivo en el control de $D$. $t a-$ bernella en Panamá dado que presentó un parasitismo muy bajo durante un seguimiento entre los años 2001 y 2002 . No obstante, estos mismos autores reconocen que en el 2001 el porcentaje de entrenudos barrenados en la zona de estudio era de $3,3 \%$, mientras que fue de $3,6 \%$ en el 2002 , lo que indicaría que una buena respuesta de este parasitoide no se daría a niveles de daño relativamente bajos y su efectividad estaría condicionada a niveles poblacionales altos del hospedante, donde las avispitas pueden hacer un mejor uso de su limitada longevidad en el estado adulto, que puede estar entre uno y cuatro días (Hernández 2010). Lo anterior es consistente con el hecho de que en general los parasitoides de larva fueron más abundantes a partir del séptimo mes de edad de la caña (Fig. 3), lo que corrobora que su presencia está altamente afectada por las poblaciones de la plaga y en virtud a la dependencia que tienen de la densidad de sus hospedantes,

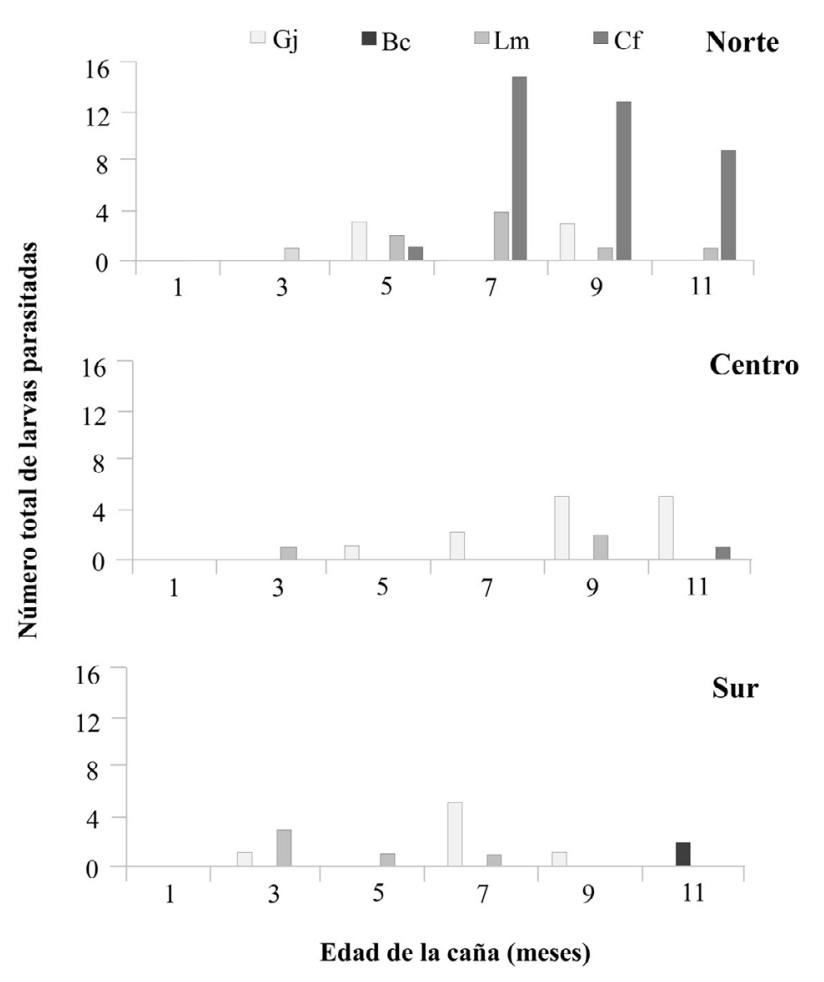

Figura 3. Número de larvas parasitadas totales en tres campos localizados en cada una de tres zonas del valle del río Cauca (Norte, Centro y Sur), por diferentes parasitoides $(\mathrm{Gj}=$ Genea jaynesi, $\mathrm{Bc}=$ Billaea claripalpis, $\mathrm{Lm}=$ Lydella minense y $\mathrm{Cf}=$ Cotesia flavipes) y durante un ciclo de cultivo de la caña de azúcar $(1-11$ meses $)$. 
lo que plantea la necesidad de revisar los planes de liberación recomendados para la región en donde no se tienen contempladas edades más avanzadas de liberación del séptimo mes de desarrollo del cultivo (Vargas 2015).

Por otra parte, existe un control biológico natural ejercido por la mosca $G$. jaynesi que, por el momento, no ha sido posible reproducirla en condiciones de laboratorio. De acuerdo con Vargas et al. (2006), el parasitismo por G. jaynesi puede llegar a ser superior a lo registrado para $L$. minense y $B$. claripalpis. En este estudio se demuestra que G. jaynesi es uno de los taquínidos que aparece en etapas fenológicas tempranas de la caña de azúcar, específicamente en la zona sur, y se encuentra distribuido en las tres zonas del valle del río Cauca a partir del quinto mes de edad de la caña. Las acciones tendientes al incremento de la acción de este parasitoide van a depender del establecimiento de prácticas de control biológico por conservación que estimulen las poblaciones y acción reguladora del insecto en los campos (Vargas et al. 2015a) mediante el cambio en los sistemas de producción de la caña de azúcar vía incorporación de mayor biodiversidad vegetal que derive en mayores servicios ecosistémicos como la mayor presencia de entomofauna benéfica (Altieri y Nicholls 2010).

Si se realiza una estimación del porcentaje de larvas parasitadas del complejo de especies de Diatraea y con relación a los diferentes parasitoides (Fig. 4), se puede decir que $G$. jaynesi y L. minense están presentes en las tres zonas, con parasitismos que no llegaron al $10 \%$. Por su parte, $C$. flavipes sólo se presentó en el norte y centro, con un mayor porcentaje en el norte, consistente con el hecho que esta es la zona de las primeras liberaciones (Vargas et al. 2015b), mientras que Billaea claripalpis solo se presentó en el sur con un parasitismo que no superó el 2 \%. Según Vargas et al. (2013) en el año 2013 L. minense fue el principal parasitoide recuperado en larvas de $D$. tabernella en la zona norte del valle del río Cauca. Sin embargo, luego de los recientes esfuerzos de liberación de Cotesia flavipes en la zona para el combate de $D$. tabernella, este estudio demuestra que C. flavipes podría tener una mayor efectividad sobre el control de esta especie (Vargas et al. 2015b).

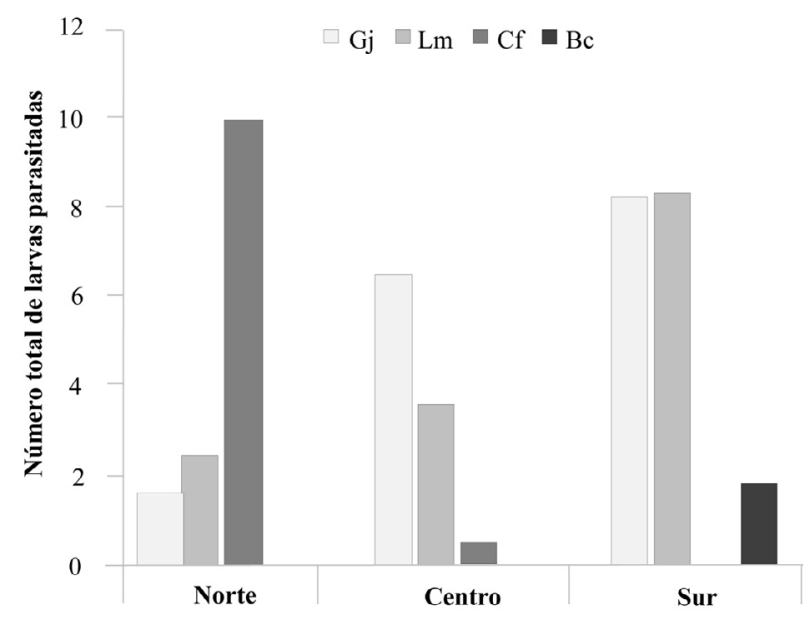

Figura 4. Porcentaje promedio de parasitismo en tres zonas del valle del río Cauca (Norte, Centro y Sur) sobre Diatraea spp. ( $\mathrm{Gj}=$ Genea jaynesi, $\mathrm{Lm}=$ Lydella minense, $\mathrm{Cf}=$ Cotesia flavipes $\mathrm{y} \mathrm{Bc}=$ Billaea claripalpis).

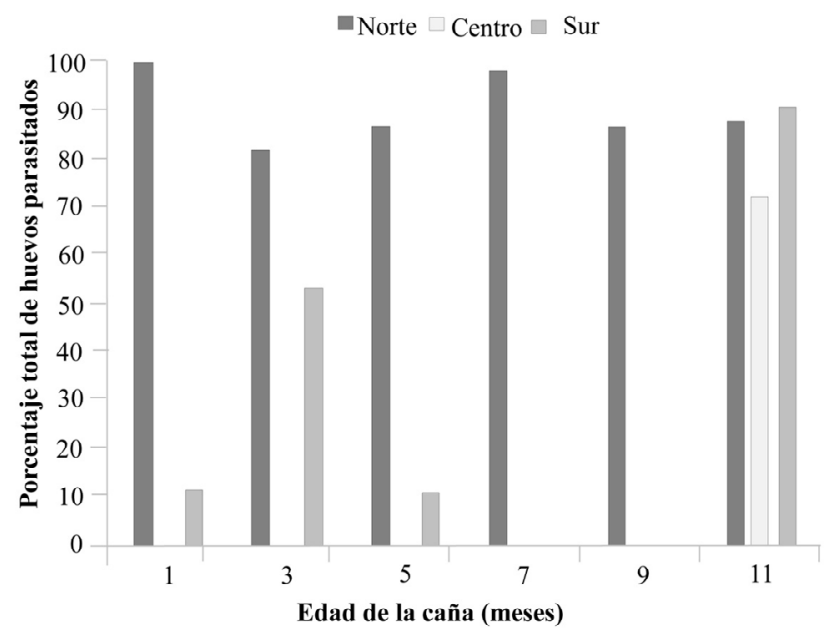

Figura 5. Porcentaje total de parasitismo de huevos por Trichogramma exiguum en tres zonas del valle del río cauca y durante un ciclo de cultivo de la caña de azúcar.

En total se recolectaron 2977 huevos en todas las zonas y épocas de evaluación, de los cuales 2142 estaban parasitados por $T$. exiguum, es decir un porcentaje de parasitismo total de $72 \%$. La Figura 5 muestra que el parasitismo en el estado de huevo es mucho más alto en la zona norte (rango entre $81,5 \%$ y $100 \%$ ), en comparación con las otras dos zonas, en donde sólo se observaron parasitismos altos hacia los 11 meses de edad de la caña (72\%, en la zona centro y $91 \%$ en la zona sur). Los porcentajes de huevos parasitados más altos en la zona norte están en concordancia con la mayor captura de huevos observados en la zona (Fig. 1B) lo que demuestra, una vez más, que la población del parasitoide está en estrecha relación con la abundancia de su hospedante en el campo. Por su parte las zonas centro y sur mostraron un parasitismo de huevos bajo, posiblemente asociado con la baja disponibilidad de huevos, lo que concuerda con lo observado por Lima y De Lima (2001), que encontraron una correlación positiva entre el número de huevos por postura encontrados en campo y el número de huevos parasitados por Trichogramma en Brasil. Vargas et al. (2015b) señalan que la acción de liberar a $T$. exiguum resultó en la reducción de hasta un $65 \%$ de las larvas de $D$. tabernella en el campo; por su parte Postali et al. (2010) comentan que la acción combinada de liberar C. flavipes y Trichogramma galloi Zucchi, en Brasil, resultó en la reducción del daño por $D$. saccharalis en un $60 \%$. La recomendación de Vargas (2015) acerca de las liberaciones T. exiguum en el valle del río Cauca señalan como límite de edad los cinco meses de desarrollo del cultivo, a la luz de los presentes resultados las liberaciones de estos parasitoides de huevos deberían de ser contempladas no sólo para prevenir la colonización de la plaga en los estados iniciales de desarrollo, sino para complementar la acción parasítica en los estados avanzados del cultivo en donde las poblaciones de huevo por la plaga se notan más altos, especialmente en la zona norte (Fig. 1B).

Daño por Diatraea spp. durante el ciclo de cultivo de la caña de azúcar. La magnitud del daño expresada en el porcentaje de tallos con daño (tallos barrenados o \% de infestación) se correlacionó de forma positiva con el incremento de la edad de la caña en las tres zonas (Norte: $\mathrm{F}=24,2 ;$ g.l. $=4$; $\mathrm{P}$ $=0,007 ; \mathrm{R}^{2}=0,85$; Centro: $\mathrm{F}=20,2 ;$ g.l. $=4 ; \mathrm{P}=0,010 ; \mathrm{R}^{2}=$ 


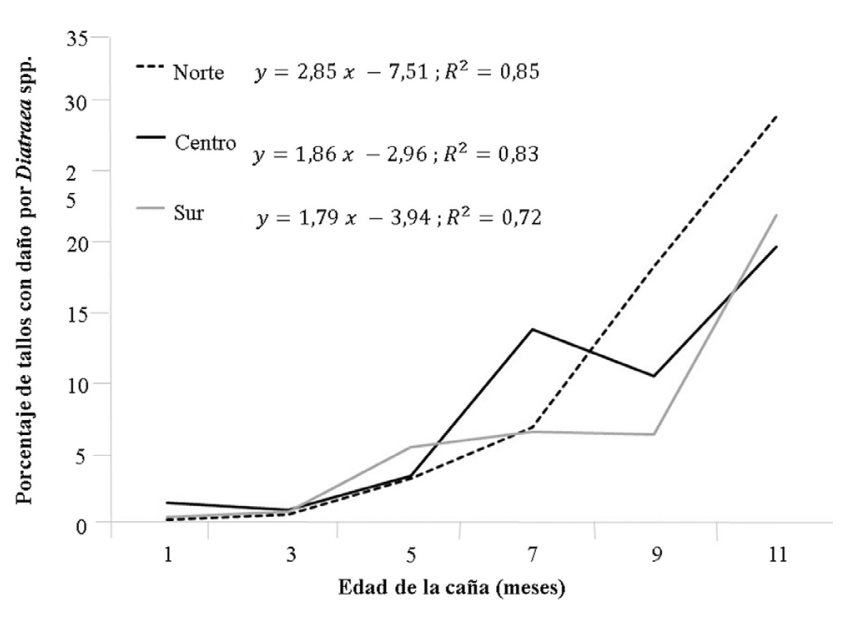

Figura 6. Porcentaje promedio de tallos con daño por Diatraea spp. en tres zonas del valle del río Cauca y durante un ciclo de cultivo de la caña de azúcar.

0,83; Sur: $\mathrm{F}=10,7$; g.1. $=4 ; \mathrm{P}=0,030 ; \mathrm{R}^{2}=0,72$ ) (Fig. 6), lo que indica que el daño por la plaga aumenta con relación a la edad del cultivo. Lo anterior ha sido comprobado desde tiempo atrás, incluso con otros barrenadores de la caña de azúcar como Eldana saccharina Walker (Lepidoptera: Pyralidae), atacando caña en Suráfrica, donde Carnegie (1974) encontró que el ataque por la plaga se recrudeció con el incremento de la edad del cultivo y fue mucho peor cuando éste estuvo sin cosecha por cerca de 16 meses o más. De igual manera Srikanth y Kurup (2011) estudiando al barrenador de la caña de azúcar en la India Chilo sacchariphagus Bojer (Lepidoptera: Crambidae) encontraron una correlación positiva entre el daño por la plaga y la edad del cultivo. De acuerdo con esto se puede proponer que independiente de la zona los planes de manejo de la plaga deben incorporar acciones de control en edades avanzadas o incluso cercanas a cosecha ya que las poblaciones adicionales que se están generando no solo constituyen daño en el presente ciclo de cultivo sino en los consecutivos y en áreas circundantes.

En cuanto al daño en los entrenudos de los tallos (intensidad de infestación o intensidad de daño), dado que era una variable que necesitaba la aparición de entrenudos en los tallos se realizó a partir de los cinco meses de edad y considerando que es una observación destructiva se hizo sólo a los 5 y 11 meses de edad tratando de no generar modificaciones en la dinámica poblacional de la plaga al interior de las cuadrículas. En todos los casos el valor de daño aumentó entre los 5 y 11 meses de edad, pero los coeficientes de variación disminuyeron a la edad de los 11 meses en aproximadamente dos veces en el caso del norte, tres veces en el caso del centro y seis veces en el caso del sur (Tabla 2). La mediana a los cinco meses tuvo un valor de cero independiente de la zona; sin embargo, se encontró una tendencia creciente hacía la zona norte, seguido del centro y, finalmente, los valores más bajos de daño en los entrenudos estuvieron en la zona sur. Esto confirma lo reportado por Gómez y Vargas (2014) en donde los coeficientes de variación del daño por la plaga fueron más altos al inicio del ciclo del cultivo con relación a las edades de maduración, es decir cerca del momento de cosecha. Estos coeficientes de variación fueron correspondientemente más bajos en la zona norte, donde el nivel de daño es más alto. De acuerdo con lo anterior se podría sugerir que las zonas centro y norte son más proclives al incremento al daño por la plaga en el valle del río Cauca si se consideran los resultados obtenidos por Vargas et al. (2005), en su diagnóstico de la situación de Diatraea spp. en la región, en donde advirtieron del crecimiento del daño en las zonas norte y centro del valle en el año 2005, en este caso asociado al incremento del daño por D. saccharalis.

Distribución espacial del porcentaje de tallos con daño (\%TD) desde el quinto al onceavo mes de edad de la caña de azúcar. La estimación del porcentaje de tallos con daño (\%TD) por Diatraea spp. en áreas no muestreadas se llevó a cabo con el método de "kriging" ordinario y la elaboración de mapas de isolíneas de daño se realizaron entre el quinto y hasta el onceavo mes de edad de la caña. Las edades de 1 y 3 meses no fueron analizadas dado que se tuvo abundancia de valores de cero que no permitieron mapear algún patrón. Por igual motivo las observaciones de la variable sólo fueron analizadas para los campos muestreados en la zona norte ya que en las zonas sur y centro la gran cantidad de valores de cero no permitieron realizar el análisis. Los mapas en la Figura 7 indican la distribución espacial del \%TD en la cuadrícula de muestreo a partir de valores estimados en los tres campos muestreados de la zona norte. Los mayores valores de \%TD se localizaron en los bordes de la cuadrícula, decreciendo progresivamente hacia el centro del cultivo. Además, se presentó una distribución agregada identificándose varios centros de conglomeración, que fueron inconsistentes en su ubicación espacial entre los 5 y 7 meses de edad, pero consistentes entre los 9 y 11 meses de edad. Lo anterior permite proponer que los adultos de Diatraea spp. ovipositaron en las plantas de caña de azúcar que encontraron en la periferia de los lotes, lo que permitió una colonización desde el borde hacia el interior en focos, inicialmente pequeños, lo que hace difícil su localización en estas edades, explicando así los altos niveles de variación en la observación del daño en los primeros meses del cultivo (Tabla 1), pero con el incremento de la edad de la caña y de la población de la plaga

Tabla 2. Porcentaje promedio de Intensidad de Infestación (II \%) por Diatraea spp. en tallos evaluados a los 5 y 11 meses de edad del cultivo de la caña de azúcar en tres zonas del valle del río Cauca.

\begin{tabular}{|c|c|c|c|c|c|c|c|c|}
\hline \multirow{2}{*}{ Zona } & \multicolumn{4}{|c|}{5 meses } & \multicolumn{4}{|c|}{11 meses } \\
\hline & Promedio & Mediana & DE & CV (\%) & Promedio & Mediana & DE & $\mathrm{CV}(\%)$ \\
\hline Norte & 4,2 & 0,0 & 9,2 & 215,6 & 6,9 & 5,1 & 6,8 & 98,7 \\
\hline Centro & 2,3 & 0,0 & 8,6 & 379,8 & 14,0 & 13,2 & 8,5 & 60,6 \\
\hline Sur & 1,2 & 0,0 & 4,1 & 338,9 & 8,5 & 8,3 & 8,1 & 96,1 \\
\hline Total & 2,6 & 0,0 & 7,7 & 300,0 & 10,2 & 9,5 & 8,4 & 83,0 \\
\hline
\end{tabular}

(II) Intensidad de Infestación: porcentaje de entrenudos barrenados. (DE) Desviación Estándar. (CV) Coeficiente de Variación. 

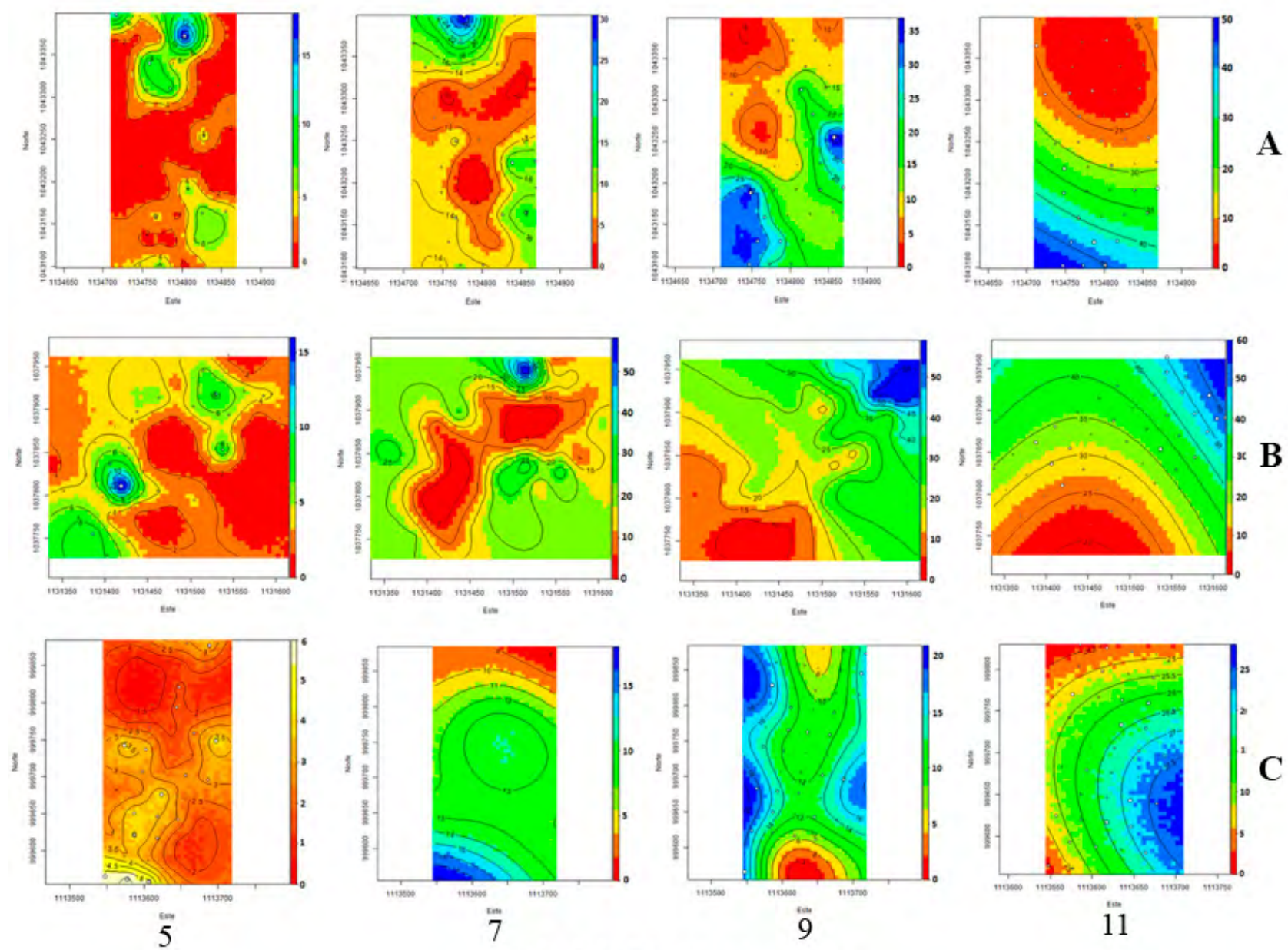

Edad de la caña (meses)

Figura 7. Mapas de distribución espacial del porcentaje de tallos dañados por Diatraea spp. entre los 5 y 11 meses de edad del cultivo de la caña de azúcar en tres campos en la zona norte del valle del río Cauca. A) Garona, B) Las Villas y C) Santa Mónica.

éstos focos se expanden disminuyendo el coeficiente de variación en la observación del daño y haciendo que su ubicación espacial sea más consistente entre el noveno y undécimo mes de la edad de la caña. Estos resultados concuerdan con otros trabajos analizando la distribución de $D$. saccharalis y en donde encontraron una distribución agregada de las larvas de la plaga y una tendencia a concentrarse en los bordes del cultivo de caña de azúcar (García et al. 2006; Costa 2009; Dinardo-Miranda et al. 2011; Santos et al. 2015), comportamiento que, con base en el presente estudio, estaría siendo compartido por las otras tres especies de la plaga. Comportamientos espaciales similares a los detectados para las poblaciones de Diatraea spp. han sido documentados para otras especies de insectos plaga, como es el caso de Jacobiasca lybica (Hemiptera: Cicadellidae) en viñedos (Ramírez et al. 2002) y Premnotrypes vorax (Coleoptera: Curculionidae) en cultivos de papa (Pérez-Álvarez et al. 2010).

\section{Conclusiones}

Diatraea tabernella se encontró sólo en la zona norte, $D$. busckella en las tres zonas, mientras que $D$. saccharalis y $D$. indigenella en el centro y sur. Las poblaciones de la plaga y los niveles de daño más altos se presentaron en la zona norte seguida, en su orden, de las zonas centro y sur. La población de huevos se hizo más notoria a partir del tercer mes de edad de la caña de azúcar y los parasitismos por Trichogramma exiguum fueron más altos en la zona norte. En las demás zonas los parasitismos se hicieron mayores hacia los 11 meses de edad. Los parasitoides de larva se evidenciaron a partir del séptimo mes, donde Cotesia flavipes presentó el mayor número de larvas parasitadas, seguida por Genea jaynesi y Lydella minense. Por su parte Billaea claripalpis se presentó sólo en la zona sur con un bajo nivel de parasitismo. Los parasitoides, tanto de larvas como de huevos, deben disponerse en el campo desde germinación, para prevenir la colonización de la plaga, y continuar en etapas avanzadas del desarrollo de la caña, donde sus poblaciones son más altas. La distribución espacial de la plaga fue agregada concentrándose en los bordes de los campos y más consistentes en su localización hacia las edades avanzadas del cultivo. Esto sugiere que la colonización de la plaga ocurre desde afuera de los lotes y que los focos de ataque se van haciendo más grandes y fáciles de observar con la edad del cultivo. Las evaluaciones y liberaciones no deben dejar de considerar los bordes de los campos, que incluso se deben priorizar en el caso de liberaciones en edades avanzadas del cultivo en donde el trabajo hacia el interior de los campos se hace difícil. 


\section{Agradecimientos}

Los autores expresan su especial agradecimiento a Álvaro Urresti, Edison Quiñones, Nelson Tipas, Orlando Rojas y Luis Bolaños del laboratorio de entomología de Cenicaña por su apoyo en las tareas de campo y laboratorio; a Yolanda Gutiérrez (Incauca), Armando González (Ingenio Mayagüez), Amanda Villegas (Ingenio Risaralda) y Carlos Rodríguez (Ingenio Sancarlos), por apoyo en la disponibilidad de los sitios de trabajo. A Cenicaña y la Universidad Autónoma de Occidente por la financiación del proyecto.

\section{Literatura citada}

ALTIERI, M. A.; NICHOLLS, C. 2010. Diseños agroecológicos para incrementar la biodiversidad de entonomofauna benéfica en agroecosistemas. Medellín: Sociedad Científica Latinoamericana de Agroecología (SOCLA). $80 \mathrm{p}$.

BADILLA, F. 2002. Un programa exitoso de control biológico de insectos plaga de la caña de azúcar en Costa Rica. Manejo Integrado de Plagas y Agroecología 64: 77-87.

CARNEGIE, A. J. M. 1974. A recrudescence of the borer Eldana saccharina Walker (Lepidoptera: Pyralididae). Proceedings of the South African Sugar Technologists' Association 15: 107110 .

COSTA, D. P. 2009. Distribuição Espacial de Diatraea saccharalis (Fabricius, 1794) (Lepidoptera: Crambidae) em Cana-deaçúcar. Universidade Federal da Grande Dourados (UFGD), Dourados, MS. Disponible en: https://tede.ufgd.edu.br/jspui/ bitstream/tede/275/1/DanielePerassaCosta.pdf. [Fecha revisión: mayo 2017].

DINARDO-MIRANDA, L. L.; FRACASSO, J. V.; PERECIN, D. 2011. Variabilidade espacial de populações de Diatraea saccharalis em canaviais e sugestão de método de amostragem. Bragantia 70: 577-585.

GALLARDO, A. 2006. Geoestadística. Ecosistemas 3: 1-11.

GARCÍA, V. M.; GONZÁLEZ, A.; GÓMEZ, L. A.; PALMA, A. E. 2006. Nuevos elementos para la determinación del daño por Diatraea spp. en caña de azúcar, pp. 179-189. En: memorias VII Congreso de la Asociación de Técnicos de la Caña de Azúcar de Colombia, Tecnicaña, 6-8 septiembre, Cali, Colombia. Tecnicaña, Cali, Colombia.

GIRALDO, R. 2002. Introducción a la Geoestadística. Teoría y Aplicación. Universidad Nacional de Colombia, Facultad de Ciencias, Departamento de Estadística, Bogotá, D. C., 97 p.

GÓMEZ, L. A.; CADENA, P. 2007. Diagnóstico de la situación de Diatraea spp. en el valle del río Cauca, segundo año. Carta Trimestral Cenicaña 29: 27-30. Disponible en: http://www.cenicana.org/pdf_privado/carta_trimestral/ct2007/ct1_07/ct1_07_ p27-30.pdf. [Fecha revisión: mayo 2017].

GÓMEZ, L. A.; DÍAZ, A. E.; LASTRA, L. A. 1996. Reconocimiento de las especies de Trichogramma asociadas con la caña de azúcar en Colombia. Revista de la Sociedad Colombiana de Entomología 22: 1-5.

GÓMEZ, L. A.; VARGAS, G. 2014. Los barrenadores de la caña de azúcar, Diatraea spp., en el valle del río Cauca: investigación participativa con énfasis en control biológico. Documento de Trabajo No. 734, Cenicaña. Disponible en: http://www.cenicana.org/pdf/no_clasificacion/6625.pdf. [Fecha revisión: mayo 2017].

HERNÁNDEZ, D. 2010. Estudio de algunos aspectos biológicos de Cotesia flavipes Cameron (Hymenoptera: Braconidae) parasitoide de Diatraea saccharalis Fabricius (Lepidoptera: Crambidae). Entomotropica 25: 69-81.

LIMA, M.; DE LIMA, J. O. G. 2001. Massas de ovos de Diatraea saccharalis (Fabr.) (Lepidoptera: Pyralidae) em cana-de-Açúcar: número de ovos e porcentagem de parasitismo por Tricho- gramma spp. (Hymenoptera: Trichogrammatidae) em condições naturais. Neotropical Entomology 30: 483-488.

LIMA, M.; GOMES, J. 2003 Diatraea saccharalis (FABR.) Em cana-de-açúcar na região norte do estado do Rio de Janeiro: flutuação populacional e parasitismo de ovos por Trichogramma ssp. Revista Universidade Rural, Série Ciências da Vida 2: 33-44.

MINITAB. 2017. MINITAB 17. Minitab, State College, PA.

OLIVER, M. A. 2013. Precision agriculture and geostatistics: How to manage agriculture more exactly. Significance 10: 17-22.

PÉREZ-ÁLVAREZ, R.; ARGÜELLES-CÁRDENAS, J.; GARRAMUÑO, E. A. 2010. Distribución espacial de Premnotrypes vo$\operatorname{rax}$ (Hustache) (Coleoptera: Curculionidae) en cultivos de papa. Corpoica Ciencia y Tecnología Agropecuaria 11: 11-20.

POSTALI, J. R.; MACHADO, P. S.; PINTO, A. 2010. Biological control of pests and a key component for sustainable sugarcane production. pp. 441-450. En: Barbosa, L. A. (Eds.). Sugarcane bioethanol R\&D for productivity and sustainability. Blucher Brazilian Science and Technology, Sao Paulo, Brasil.

R CORE TEAM. 2013. R: A language and environment for statistical computing. R Foundation for Statistical Computing, Vienna, Austria. URL http://www.R-project.org/.

RAMÍREZ, J.; GONZÁLEZ, J.; OCETE, R.; LÓPEZ, M. 2002. Descripción geoestadística de la distribución espacial de los huevos del mosquito verde Jacobiasca lybica (Bergenin \& Zanon) (Homoptera: Cicadellidae) en viñedo: modelización y mapeo. Boletín de Sanidad Vegetal Plagas 28: 87-95.

RODRÍGUEZ, V.; CHAVARRİA, L.; GÓMEZ, I., PEÑALOZA, Y.; TEJADA, M. 2004. Desarrollo del parasitoide Cotesia flavipes Cámeron, 1891 (Hymenoptera: Braconidae) en Diatraea tabernella Dyar y Diatraea saccharalis Fabricius, 1794 (Lepidoptera: Pyralidae), y su efectividad en el control de Diatraea tabernella. Tecnociencia 6: 85-94.

SALAZAR, J.; OVIEDO, R.; SÁENZ, C. 2003. Descripcion, manejo y control del barrenador común del tallo de la caña de azucar en Costa Rica. San José: Dirección de Investigación y Extensión de la Caña de Azúcar. Disponible en: http://www.laica.co.cr/ biblioteca/servlet/DownloadServlet?c $=443 \& \mathrm{~s}=1774 \& d=9707$. [Fecha revisión: mayo 2017].

SANTOS, E. A.; STAKE, R. F.; MACHADO, M. R. R.; MAGANO, D. A.; GUEDES, J. V. C. 2015. Distribuição espaço-temporal de Diatraea saccharalis sobre cana-de-açúcar em são Vicente do Sul-RS., pp. 1-6. En: IV Simpósio de Geoestatística Aplicada em Ciências Agrárias. Sao Paulo. Disponible en: http://www. bibliotekevirtual.org/simposios/SGEA/SGEA-2015/manejo-deculturas/59.pdf. [Fecha revisión: mayo 2017].

SOLIS M. A.; METZ A. M. 2016. An illustrated guide to the identification of the known species of Diatraea guilding (Lepidoptera, Crambidae, Crambinae) based on genitalia. Zookeys 565: 73-121.

SRIKANTH, J.; KURUP, N. K. 2011. Damage pattern of sugarcane internode borer Chilo sacchariphagus indicus (Kapur) in Tamil Nadu State, southern India. International Sugar Journal 113: 590-594.

TREJOS, J. A.; LONDOÑO, F.; GÓMEZ, L. A.; RAIGOSA, J. D. 1986. Distribución de las especies de Diatraea (Pyralidae) en caña de azúcar (Saccharum sp.) en el valle geográfico del río Cauca y algunas observaciones sobre su parasitismo. Acta Agronómica 36: 59-68.

VARGAS, G. 2015. Retos y oportunidades en el manejo de los barrenadores del tallo, Diatraea spp. Cenicaña, Serie Divulgativa No. 17, 6 p. Disponible en: http://www.cenicana.org/pdf/serie_divulgativa/sd_17/sd_17.pdf. [Fecha revisión: mayo 2017].

VARGĀS, G. A.; OBĀNDO, V.; GÓMEZ, L. A. 2005. Diagnóstico de la situación de Diatraea spp. en el valle del río Cauca. Carta Trimestral Cenicaña 27: 27-32. Disponible en: http://www. cenicana.org/publicaciones/carta_trimestral/ct2005/ct3y4_05/ ct3y4_05_p27-31.php. [Fecha revisión: mayo 2017].

VARGAS, G.; OBANDO, V.; GÓMEZ, L. A. 2006. Jaynesleskia jaynesi: otra alternativa para el manejo de Diatraea spp. Carta 
Trimestral Cenicaña 28: 3-5. Disponible en: http://www.cenicana.org/publicaciones/carta_trimestral/ct2006/ct2_06/ct2_06_ p3-5.php. [Fecha revisión: mayo 2017].

VARGAS, G.; LASTRA, L. A.; SOLÍS, M. A. 2013. First record of Diatraea tabernella (Lepidoptera: Crambidae) in the Cauca River Valley of Colombia. Florida Entomologist 96: 1198-1201.

VARGAS G.; GÓMEZ, L. A.; MICHAUD, J. P. 2015a. Sugarcane stem borers of the Colombian Cauca River Valley: pest status, biology and control. Florida Entomologist 98: 728-735.

VARGAS, G.; VILLEGAS, A.; RAMÍREZ, D.; BARCO, L. E.; GUTIÉRREZ, Y.; HERRERA, D.; VALENCIA, I. C. 2015 b. Eficacia de las liberaciones de enemigos naturales para el manejo de Diatraea tabernella y D. busckella en la zona norte del valle del río Cauca. pp. 411-422. En: Memorias X congreso de la Asociación Colombiana de Técnicos de la Caña de Azúcar, TECNICAÑA. Cali, Colombia Septiembre 14-18.

VARGAS, G. A.; LASTRA, L. A.; RAMÍREZ, G. D.; SOLIS, A. 2018. The Diatraea complex (Lepidoptera: Crambidae) in
Colombia's Cauca river valley: making a case for the geographically localized approach. Neotropical Entomology 47: 395-402. WEBSTER, R.; OLIVER, M. 2007. Geostatistics for Environmental Scientists. Londres: Jonh Wiley \& Sons Ltd., 2007. 333 p.

Recibido: 11-jun-2017 • Aceptado: 07-may-2018

Citación sugerida:

LEYTON-FLOR, S. A.; GORDILLO, M.; GONZÁLEZ, P. A.; OSPINA, J. A.; VARGAS, G. 2018. Distribución espacio-temporal de Diatraea spp. (Lepidoptera: Crambidae) en el valle del río Cauca, Colombia. Revista Colombiana de Entomología 44 (2): 177-186. Julio - Diciembre 2018. 